\title{
On the application of optical forward-scattering to bacterial identification in an automated clinical analysis perspective
}

\author{
Umberto Minoni ${ }^{1}$, Alberto Signoroni ${ }^{1}$, and Giulia Nassini ${ }^{1}$ \\ ${ }^{1}$ Department of Information Engineering, University of Brescia, \\ Via Branze 38, I-25133 Brescia, Italy
}

\section{Corresponding author}

Umberto Minoni, Department of Information Engineering, University of Brescia, Via Branze 38, I-25123 Brescia, Italy e-mail: umberto.minoni@unibs.it tel: (+39) 0303715442 fax: (+39) 030380014

\section{Abstract}

The Optical Forward Scattering (OFS) technique can be used to identify pathogens by direct observation of bacteria colonies growing on a culture plate. The identification is based on the acquisition of scattering images from isolated colonies and their subsequent comparison with reference images acquired from known bacteria. The technique has been mainly studied for the identification of pathogens in the food-safety field. This paper focuses on the possibility of extending the applicability of the technique to the field of clinical laboratory automation. This scenario requires that the paradigm of image acquisition at fixed colony-dimension, well established in the food-safety applications, should be substituted by an acquisition at fixed incubation time. As a consequence, the scatterometer must be adjustable in real-time for adapting to the actual features of the bacterial colony. The paper describes an OFS system prototype qualified by the possibility to tune both the laser beam diameter and the acquisition camera field of view.

Preliminary experiments on bacteria cultures from pathogens causing infections of the urinary tract show that the proposed approach is promising for the development of an 
automated bacteria identification station. The new OFS approach also involves an alternative method for building a reference image database for subsequent image analysis.

Keywords: bacterial rapid identification; colonies analysis; light scattering.

\section{Introduction}

Rapid pathogenic bacteria identification is crucial in clinical settings, especially where their fast recognition has a vital impact on patient care and epidemics control. In order to reduce the turnaround time to complete the clinical analysis and also to face the ubiquitous budget-cut challenge, the clinical laboratories are now more often considering the installation of automated stations. There are systems based on inoculation on plates and cards that, after incubation, can be automatically read to identify a number of micro-organisms (e.g., VITEK by BioMérieux, Phoenix by BD, MicroScan by Siemens). Significant is the turnaround time for these solutions as well as the cost of the required consumables. The matrix-assisted laser desorption ionization time-of-flight mass spectrometry (MALDI-TOF MS) is more and more often proposed as a rapid and less expensive method identifying isolated bacteria (Seng et al., 2009). The MALDI-TOF mass spectrometry, compared to the traditional identification methods, has the advantages of faster response time and simpler usage (Risch et al., 2010). Nevertheless, the MALDI-TOF MS approach seems not yet to be definitive, because it cannot provide a complete bacteria species discrimination and in general it is not sensitive to species variations (serotypes). Moreover, systems based on this technique are not yet currently approved by the US Food and Drug Administration (Patel, 2013). In the perspective of the full automation of a clinical microbiology laboratory, the advent of liquid based microbiology (LBM) plays a key role by simplifying the specimen workflow, resulting in a remarkable reduction of the turnaround time and in an easier sampling, transport, and storage. LBM automated stations typically integrate processing of high definition culture images providing the microbiologist with software tools helping the analysis work. To better exploit the image processing system it is also currently investigated the use of plate illumination extending beyond the visible spectrum (Yoon et al., 2010, 2013). In some restricted cases, the image processing can even provide a reliable bacteria identification, nevertheless this approach cannot be applied to a significant number of pathogens. The optical forward-scattering (OFS) 
technique, that has been proposed as a label-free method for bacterial identification (Banada et al., 2007, 2009; Bae et al. 2011b; Suchwalko et al. 2013) is also based on image processing, but in this case the identification is done at the colony level and, under suitable conditions, it has been demonstrated that the colony can provide a reliable signature of the constituting bacterium at the serotype level (Rajwa et al. 2006; Akova et al. 2010; Buzalewicz et al., 2011). The OFS technique has been mainly investigated in the foodsafety field, however, due to its great potential its usage also in the clinical laboratory is worth to be investigated. Differing from the food-safety context, the analysis in a clinical laboratory undergoes a wider variability due to the higher number of bacterial species of interest, the possible simultaneous presence of several species in the same sample, and the fact that the bacterial load can spread over a wide range according to the kind of biological sample and to the infection level. These peculiarities give rise to a series of issues to be addressed for a possible application and exploitation of OFS technologies in a clinical application scenario. This motivated our work and suggested the solutions we documented and experimented in this work.

\section{Clinical analysis and laboratory automation}

After collection, clinical specimens arrive in the microbiological laboratory in the form of liquid samples contained in closed plastic tubes. The homogenous nature of the liquid specimens is essential for the automation of the plate inoculation. Modern laboratory automation technologies make it possible to automate the pre-analytical phases of the analysis process. In particular, specimen processing, planting and streaking, Gram slide preparation, and enrichment broth inoculation can be robotized. The use of barcodes on both the specimen and the culture plate ensures the traceability of the analysis and the correct patient connection. The plates automatically prepared can then be placed in automatic aerobic or anaerobic incubators. The automation under the supervision of a controlling computer makes possible the application of streaking and growing programs specific for each culture plate. Manufacturers of the automation systems have recently introduced also digital imaging tools aimed to monitor bacteria growth and to provide the microbiologist with high definition images of the cultures. More and more sophisticated algorithms are implemented to pre-process the culture images eventually acquired with innovative image acquisition technologies giving the biologist an important aid. Recent reviews about the current technologies and trends in clinical 
laboratory automation are given in Novak et al. (2013) and in Bourbeau and Ledeboer (2013).

\section{OFS for clinical domain applications: main issues and proposed solutions}

The main constraint in the actual implementation of the OFS technique is the need to analyse colonies with a fixed dimension by using a laser beam having a diameter almost coincident, at a relatively small tolerance, to that of the isolated colony under test (Banada et al., 2009; Gong et al., 2013; Bae et al., 2009). Complying with the fixed diameter condition in an automated clinical laboratory becomes not compatible with the plate processing workflow: due to the great inherent variability of the input samples (both in terms of species and bacterial load), the right culture duration corresponding to the right colony size can result in an ill-posed problem and, in general, it cannot be predetermined. At the same time, a frequent verification of the colony diameter on each plate inside the automated incubator is not feasible without introducing system inefficiencies and possible impairment of the growth process. A possible solution, compatible with the requirements arising from an automated plant workflow, is to release the diameter constraint giving the possibility to take the scattering patterns from colonies having significant diameter variability. In order to guarantee an ideal, uniform and valid colony OFS acquisition setup, and to produce data that hopefully are still comparable with others, we propose and implement the tuning of the laser beam diameter to the colony size before taking the scattering picture. This added flexibility generates the additional possibility to define different colony dimension working ranges, aimed to maintain, or possibly improve, the bacteria discrimination capabilities of an OFS systems. To further optimize the image acquisition with respect to the variability of the scattering angle, caused by both the different bacteria and by the different colony sizes, we also introduce the possibility to adjust the distance of the digital camera used for the image acquisition from the Petri dish.

For this pilot study pathogens of the urinary tract infection (UTI) have been considered because of the wide diffusion of UTIs and to the great potential advantages coming from the immediate and non-destructive identification of these pathogens at the plate level. 


\section{Materials and methods}

\subsection{System prototype description}

Following the considerations so far presented, we designed and realized a prototype of scatterometer suitable for the experimental activity. The scheme of the instrument is depicted in Fig. 1. Although the implemented prototype is manually operated, the architecture of the measuring device can easily be adapted to work in a completely automated way. Referring to Fig. 1, the Petri dish taken from the incubator is positioned on the measuring plate and with the help of the vision sub-system including the ring illuminator (RI) and the imaging camera $\mathrm{C} 1$ it is precisely moved in the $\mathrm{x}, \mathrm{y}$ plane until the desired colony is centered with the axis of the illuminating laser beam. Once the colony is in place, the scattering pattern is acquired by the high-definition camera C2. The prototype has the possibility of adjusting the laser beam diameter $\phi_{\mathrm{LB}}$, by means of the focusing lens (FL), in a range from 0.1 up to $5 \mathrm{~mm}$. Another important adjustment at our disposal is the possibility of changing the distance $\mathrm{d}_{\mathrm{sc}}$ of the scattering camera from the measuring plane. This feature is used to tune the system to the scattering angle; the wider the angle the closer the camera to the colony.
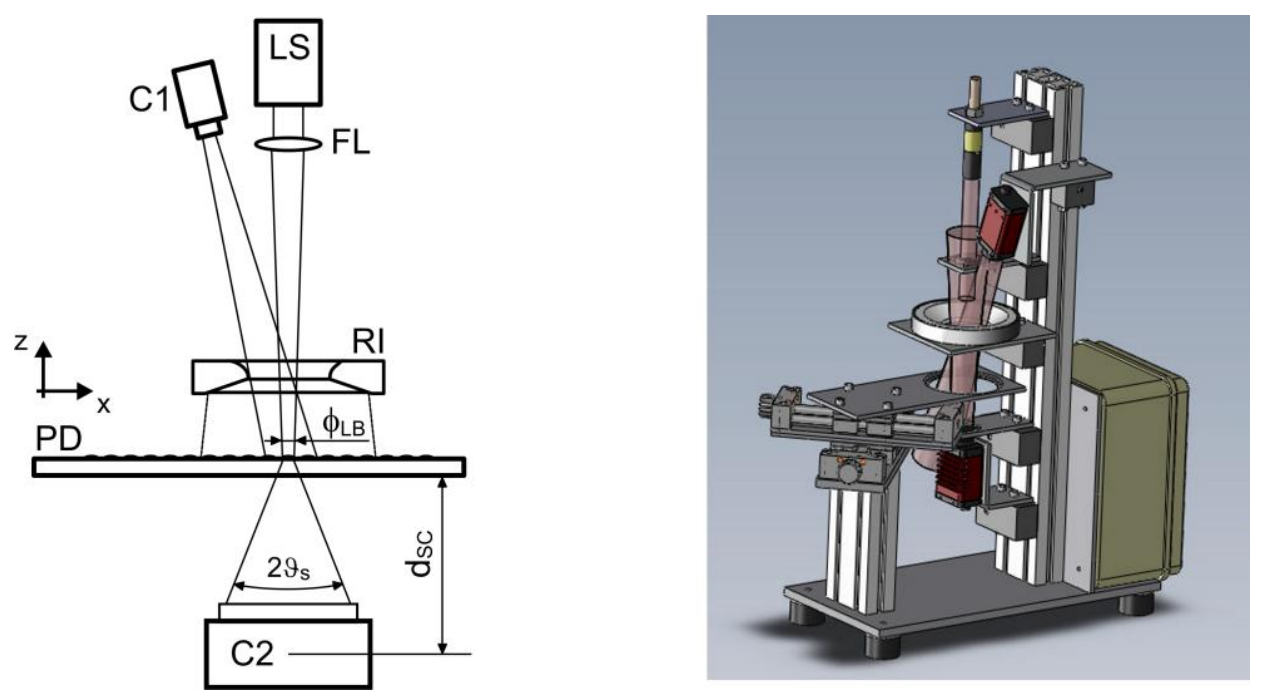

Figure 1. The scatterometer used for the experiments. LS: laser source; FL: focusing lens; PD: Petri's dish; RI: ring illuminator; C1: imaging camera; C2: scattering pattern camera; $\phi_{\mathrm{LB}}$ : adjustable laser beam diameter; $\vartheta_{\mathrm{s}}$ : scattering angle; $d_{s c:}$ adjustable scattering camera distance.

\subsection{Sample preparation}

The most common UTI pathogens have been considered: Streptococcus agalactiae, Staphylococcus aureus, Staphylococcus saprophyticus, Proteus mirabilis, Escherichia coli 
and Enterococcus faecalis. In addition, we used three common bacteria belonging to the Enterobacteriaceae family: Klebsiella pneumoniae, Salmonella typhimurium and Yersinia enterocolitica. All the bacteria species studied during the experimental activity are from ATCC ${ }^{\circledR}$ strains. The UTI pathogens have been cultured on chromogenic culture media (CHROMAgar by BD), the Enterobacteriaceae family bacteria have been cultured on Mueller Hinton II agar plates from two different manufacturers (BD and Biomérieux). The cultures have been prepared starting from a 0.5 McFarland bacterial suspension diluted in order to obtain about 50 to 100 colonies per plate. Two well-known procedures can be used to prepare the cultures: i) inoculation with a micro-pipette, ii) spreading or streaking with a calibrated loop. Provided that the bacterial load of the suspension is well controlled, the cultures prepared by micro-pipette inoculation are characterized by uniformly distributed colonies having the same dimension. The condition of a controlled bacterial load is not satisfied in a clinical case due to the unknown status of the biological samples. Indeed, the estimation of the bacterial load can be helpful to understand the degree of patient infection. Therefore, the best solution is to pick up the suspension with a calibrated loop and streak it on the plate through a defined pattern.

After the inoculation of the suspension on the agar medium the cultures were incubated at $37^{\circ} \mathrm{C}$ for 16 hours. If not differently specified these culturing conditions were applied for all the experiments described in the following.

\subsection{Image acquisition}

The scattering pattern images are acquired with the system of Fig. 1. First, the Petri's dish with the grown colonies is positioned on the holding plate where the laser beam used to generate the scattering patterns is intercepted by the culture agar. We implemented an acquisition setup and a GUI interface where the images from the cameras are streamed on the monitor of the controlling computer. The video stream provided by the first camera $\mathrm{C} 1$ shows the colonies on the dish, whereas the second video stream from camera C2 shows the light scattered through the dish. Camera C1 can be adjusted in order to frame a selected zone of the dish around the light shot produced by the collimated laser beam. Looking at the real-time image of the plate surface and by moving the culture dish on the XY plane it is possible to align a single colony to the laser beam. To optimize the image acquisition, the illuminator distance from the plate and the 
emitted light intensity can be adjusted. The second camera C2 acquiring the scattering pattern can be adjusted along the $\mathrm{Z}$ axis to obtain the best imaging condition for the investigated colony. The definitive system setup is obtained looking at the scattering pattern images provided by camera $\mathrm{C} 2$, both the plate position and the laser beam diameter are adjusted to obtain the best image.

Following the plate positioning and image optimization, a picture of the whole plate is stored as a reference image that is useful to trace the bacteria colony and the generated forward-scattering images. In particular, a post-processing of the reference images can be used to compare the macroscopic characteristics of the investigated colonies such as dimension, contours shape with the scattering pattern features.

At the end, the scattering pattern image is stored and the corresponding bacterial colony is tagged on the reference plate image. For this preliminary study on the applicability of the forward-scattering technique to the clinical field, the scattering patterns features have been collected from a suitable number of samples (dozens patterns for each species) and visually analysed. This approach is adequate to draw the first and more important considerations about the technology suitability and expected effectivness in the foreseen application field.

\subsection{The fixed growing time condition}

As previously stated, in the automated clinical analysis field we have to assume that the examined bacteria species are unknown and, consequently, also the incubation time necessary to reach a given colony diameter is unknown. Nevertheless our approach requires to pre-set in advance the incubation time. The choice of the incubation period must take into account that, as well known, the bacteria growth is not linear and that the best scattering patterns are acquired during the exponential growing phase. Provided that the duration of the growing phases is specific to each culture (bacterium and medium combination) nevertheless we can find a compromise incubation time suitable for a number of different bacteria cultured on the same plate. In view of lab automation, it is also reasonable to consider two or (max) three subsequent image acquisitions at different incubation time intervals enhancing the chance to get images from colonies in the optimal growing phase. In our experiments, if not otherwise specified, and inspired by good laboratory practice, the images were acquired at 16 hours of incubation, 
obtaining colonies with a diameter ranging from 0.5 to $2.5 \mathrm{~mm}$ for the envisaged bacteria.

\section{Experimental results}

\subsection{Reproducibility verification}

The first experiments were done to visually verify the capability of the prototype to provide reproducible scattering pattern images from different colonies of the same species sampled on the same plate. Figure 2 shows an example of the scattering patterns acquired for 5 different species of bacteria. Pattern images from 5 randomly selected colonies for each plate are grouped in each row. It can be noted that, for each species, the patterns appear to be very similar, so evidencing a good reproducibility of the acquired images; the patterns had the same scattering angle and the same main features. In a second series of observations we analized plates filled with the same media and compared the scattering images formed by colonies having similar dimensions but from different plates. The inter-plate comparison evidenced the very same degree of reproducibility of the the intra-plate case. 


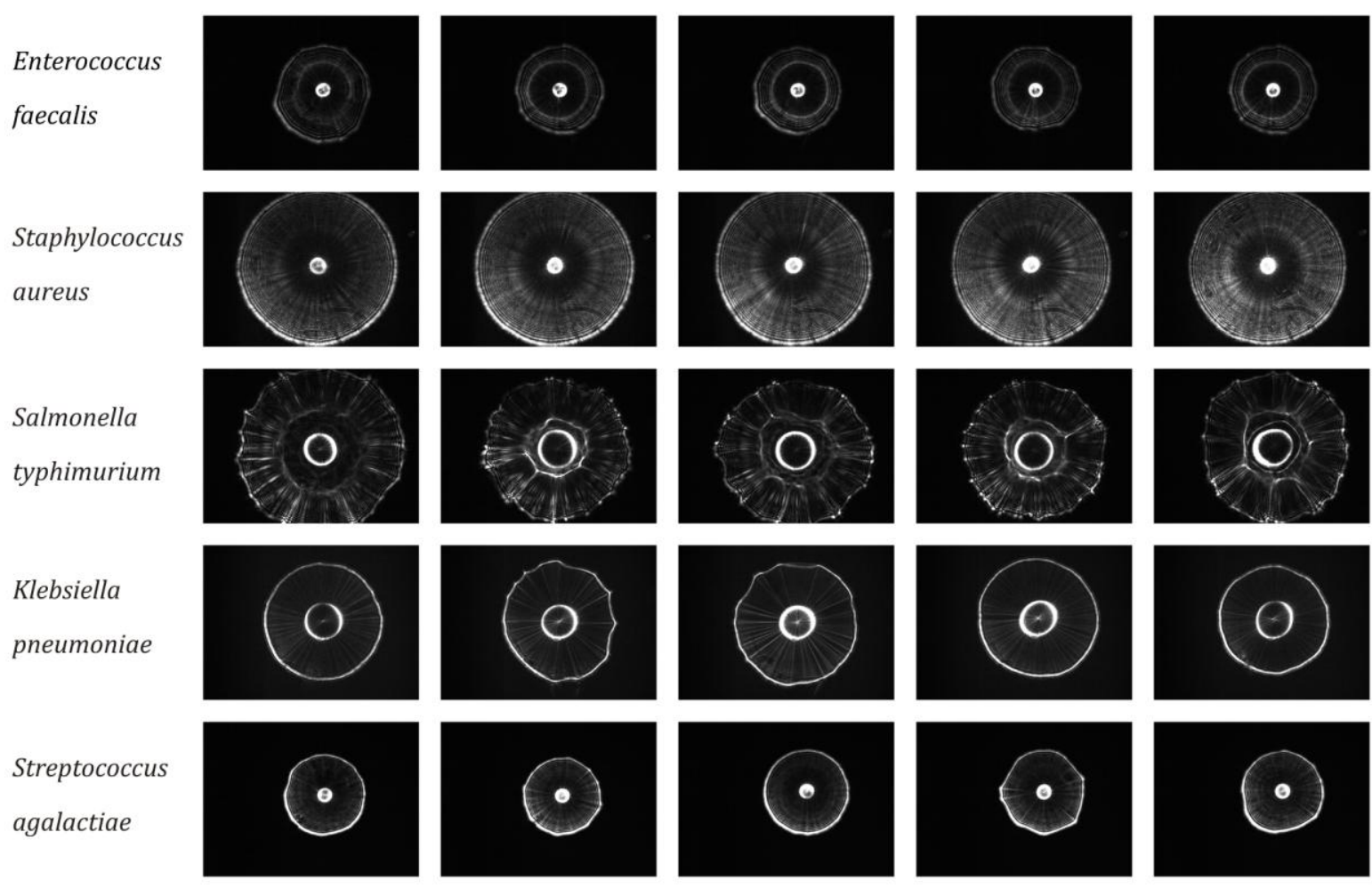

Figure 2. Comparison of scattering patterns from various colonies of different bacteria. The patterns in a row come from colonies on the same plate. All the species were plated on Mueller Hinton II - BD agar plate.

\subsection{Medium and time variability}

Another experimental session was dedicated to test the influence of the agar nature on the scattering patterns. As reported in Bae et al. (2011a) the agar type can deeply influence the scattering pattern, this is coherent with the fact that the agar is the nourishment of the colony and therefore it regulates the colony formation in terms of growth rate and, in case of chromogenic agar, in terms of pigmentation. The changes of agar concentration and nutrients are sufficient to influence the scattering patterns, consequently, since the agar recipe changes with the producer it was important to investigate how much the scattering pattern depends on the agar manufacturer. In addition, since the laser light path includes the agar medium, the agar itself can be considered a reason of scattering image variations. 
Biomérieux

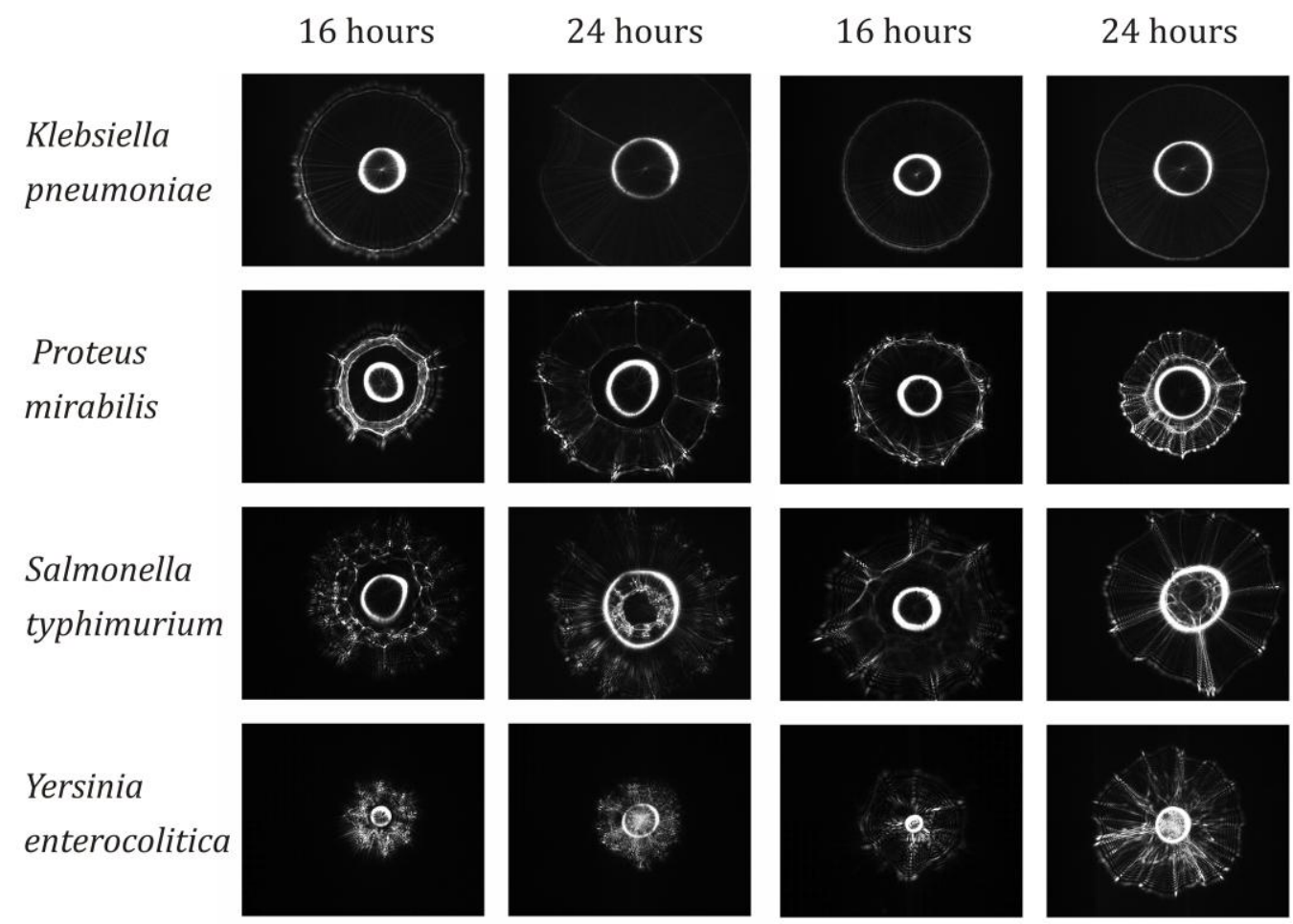

Figure 3. Comparison of the scattering patterns from colonies grown on Muller Hinton II agar by different producers (Biomérieux and BD). The plates were incubated at $37^{\circ}$ for an incubation time of 16 hours or of 24 hours.

The comparison was performed with all the bacteria listed in Sec. 2.2 with the standard 16 hours and prolonged 24 hours incubation. By observing different cultures we realized that the colonies grown on the Biomérieux medium showed an average growth rate about $40 \%$ greater than that on the BD medium. As an example, in the case of Staphylococcus aureus at 16 hours incubation, the colonies cultured on Biomérieux medium had a diameter ranging from 1.2 to $1.5 \mathrm{~mm}$ whereas colonies on BD medium showed a diameter varying from 0.7 to $0.9 \mathrm{~mm}$. A typical result is shown in Fig. 3 where the comparison among cultures grown on two media is presented. According to the procedure that will be described later, for the scattering pattern acquisition the laser beam diameter was adapted to the colony diameter. As detailed in the next section, we expect different patterns from colonies incubated for different time intervals; here we can also see differences of the patterns obtained from the two media at the same incubation time interval of both 16 hours and 24 hours, thus confirming a significant dependence of the scattering patterns from the agar composition. From Fig. 3 the 
significant scattering pattern changes due to different incubation times are evident for the all species.

\subsection{The colony dimension variability at fixed time}

For a given culture setup some bacteria such as the Staphylococcus aureus do not present a significant intra-plate colony dimension variability, others such as the Proteus mirabilis give rise to a great intra-plate dimension variability.

Table I. Colony diameter and colony diffraction angle of different bacterial species cultured on Mueller Hinton II agar for 16 hours.

\begin{tabular}{|l|c|c|}
\hline Bacterial species & Colony diameter $(\mathrm{mm})$ & Scattering angle (deg) \\
\hline Yersinia enterocolitica & $0.5-1.1$ & $3-4.9$ \\
\hline Enterococcus faecalis & $0.8-1$ & $12.5-8.3$ \\
\hline Streptococcus agalactiae & $0.8-1$ & $7.2-8.1$ \\
\hline Staphylococcus aureus & 1 & $14.5-16.4$ \\
\hline Proteus mirabilis & $1.7-2.2$ & $5.6-8.2$ \\
\hline Klebsiella pneumoniae & $1.9-2.4$ & $11.2-11.6$ \\
\hline Salmonella typhimurium & $2-2.5$ & $5.6-6.9$ \\
\hline Escherichia coli & $1.8-2.8$ & $4.8-6.3$ \\
\hline
\end{tabular}

The reason is probably related to different growing rate. When the bacterium has a slow growing rate, at the end of the standard 16 hours incubation, all the colonies on the plate are still small and well separated, thus, there is no real competition for nourishment. In contrast, when the growing rate is higher we can find colonies having considerably different diameters on the same plate. The colony dimension variability is clearly shown in Tab. I (second column) were the data refers to cultures grown on Mueller Hinton II agar with an incubation time equal to 16 hours. We can see that there is significant diameter variability both among the colonies of the same species and among colonies of diverse species. The case of Staphylococcus aureus can be considered an exception where we observed an almost constant diameter of $1 \mathrm{~mm}$. 

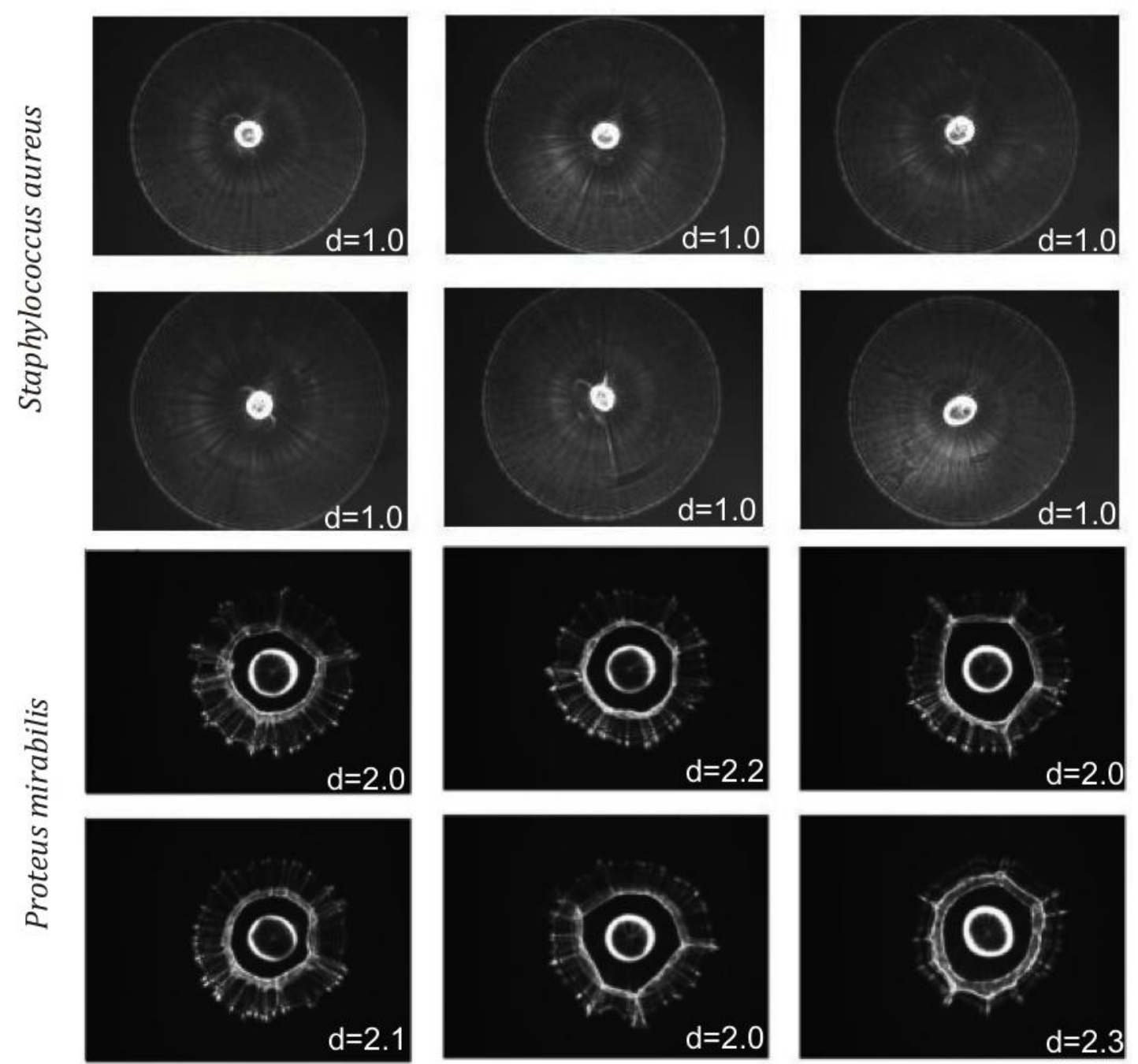

Figure 4. Comparison of scattering patterns from colonies having different dimensions. The average colony diameter is indicated as d. The media was Mueller Hinton II by BD, the incubation time of 16 hours at $37^{\circ} \mathrm{C}$.

Figure 4 shows, as a representative example of the influence of the dimension variability, the scattering patterns of six colonies of Staphylococcus aureus and six of Proteus mirabilis, the images have been acquired after the laser beam diameter was adjusted to match the dimension of each colony. We can note that, as expected due to the diameter invariability of the colonies from Staphylococcus aureus, the patterns corresponding to this bacterium are much more similar to each other with respect to those of the Proteus mirabilis. This is a confirmation of the fact that the colony dimension can play a key role in the definition of the scattering pattern. Nevertheless, good similarities persist among the patterns provided by the Proteus mirabilis with greater and evident differentiation of these patterns with respect to those of Staphylococcus aureus. In this, as well as in all the other examined cases, appropriate machine learning techniques can then be successfully used to differentiate, with good confidence, the bacterial species. In other 
words, the diameter variability can introduce variations of the scattering pattern that are significantly dependent on the species we are looking at, however, from our evidences, this does not appear to be a serious impediment to the ability of differentiating the bacterial species, especially by using a machine learning algorithm that can rely on the visual features that remain statistically unchanged (e.g. captured by tools as Zernike moments (Khotanzad and Hong, 1990; Banada et al., 2007)).

\subsection{Scattering angle and camera distance adjusting}

Light propagation through a bacterial colony cannot be accurately described without the knowledge of the colony morphology, and the optical properties of both the bacteria agglomerates and extracellular materials. A detailed model of the bacterial colony is given in Bae et al. $(2007,2010)$ where the characterizing parameters of the scattering patterns are correlated to the colony height and diameter. From the model it appears clear how the scattering angle undergoes to significant variations when either the thickness or the diameter of the colony changes. In particular, for a fixed colony diameter the scattering angle increases with the center thickness of the colony and for a fixed thickness the scattering angle is inversely proportional to the colony diameter. Assuming a Gaussian profile of the colony cross-section, the maximum scattering angle relates with the colony diameter in a quite complex way.

As an example, Table I (third column) shows the scattering angles of the investigated colonies. As seen for the diameter, also for the scattering angle, we can note a remarkable variability among colonies of the same species and, more significantly, among colonies of different species. 

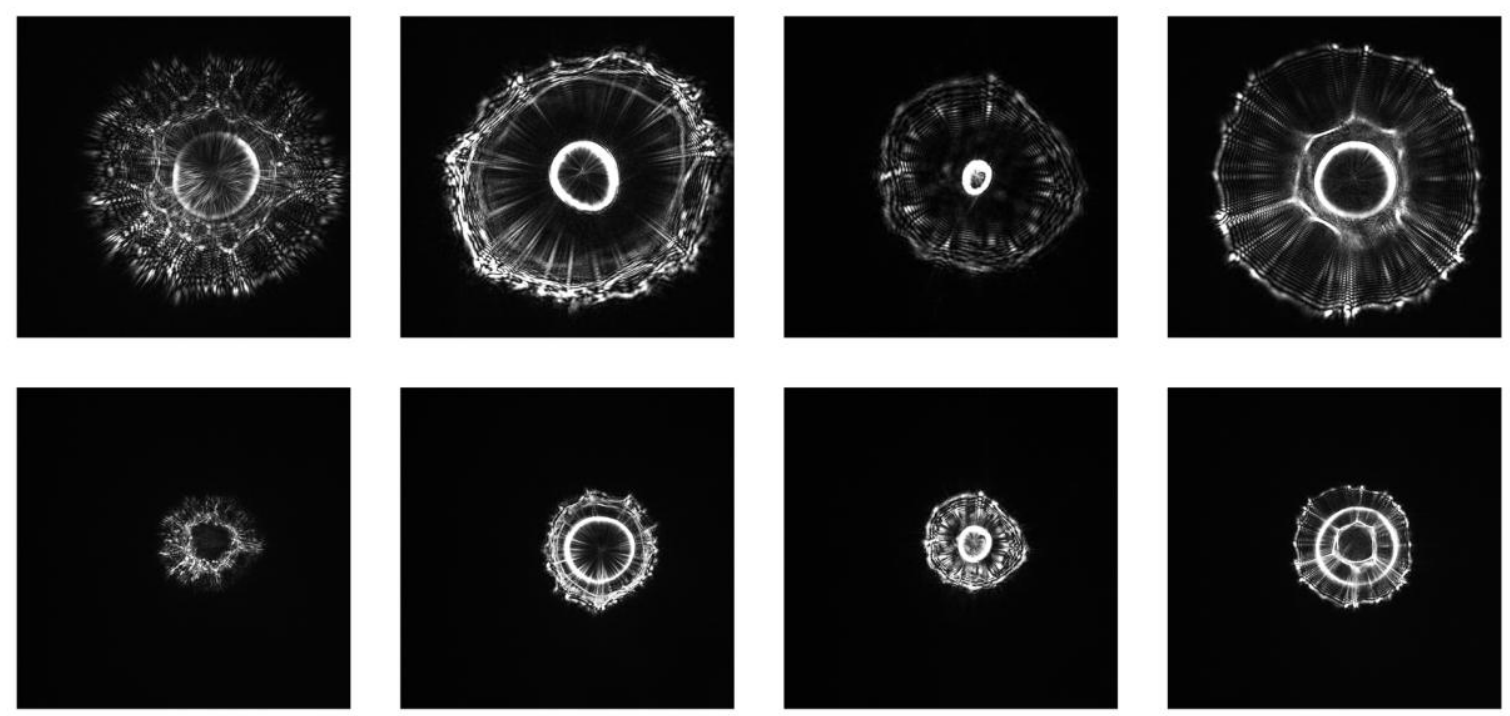

Figure 5. Example of scattering patterns acquired at different distances between the camera and the Petri Dish. In the first row the images acquired by the camera at a distance of $20 \mathrm{~mm}$, in the second row the images acquired when the camera is very close to the culture plate. In each column different bacteria species cultured on Mueller Hinton II by BD for 16 hours of incubation at $37^{\circ}$, from the left: Escherichia coli, Proteus mirabilis , Yersinia enterocolitica, Salmonella typhimurium.

The scattering angle has a great relevance in the scattering pattern formation, in particular it set the optimal distance to put the camera in order to frame the more informative pattern. In other words, neither the very near field nor the far field scattering images can be considered sufficiently informative about the colony structure and therefore suitable for colony identification. To get good scattering patterns from the different colonies it is therefore straightforward to foresee the possibility of adjusting the field of view of the camera according to the colony scattering angle and colony diameter. Referring to Fig. 1, the field of view adjustment can be obtained by changing the camera distance from the media plate. The camera positioning let us optimize the image acquisition by framing the significant part of the scattering pattern with the whole camera sensor obtaining more detailed pattern images. As an example, in Fig. 5 we can see, for different bacteria species, the scattering pattern images of the same colony at different camera distances. All pictures are characterized by a brighter central spot that correspond to the projection of the colony illuminated by the laser. The best-detailed pattern has been acquired by posing the camera at a distance producing an image covering the entire active area of the image sensor. For the set of the analyzed bacteria two suitable camera distances are found: camera very close to the culture plate and 
camera at a distance of $20 \mathrm{~mm}$. The images are acquired with the camera close to the plate in case of large scattering angles, whilst the camera is moved away in case of small scattering angles. In the perspective of the automated scattering station, there is also the possibility to implement, as a standard approach, the acquisition of scattering pattern sequences from shots taken at different distances. The analysis of the pattern evolution as a function of the camera distance could add some information that can be useful for the identification.

\section{Discussion}

Before starting with the investigation so far described, a screening of the current routines applied by a number of laboratories involved in the treatment of the specimen for bacterial cultures has been carried out. We have seen that the labs using the most advanced technique for the automatic bacterial culture could benefit from an integrated automatic bacteria recognition working directly on the culture plate. The forwardscattering technique has the potential for the implementation of an identification unit which can easily be integrated in the plate processing workflow. The automated clinical analysis, however, imposes some sample processing constraints which require an identification unit capable of adapting itself to the great variability of the bacteria colonies in terms of different species, dimensions, and agar substrates. In our view, to meet the requirements, the forward-scattering technique must be implemented releasing the restriction of the fixed colony diameter so far proposed as a standard condition in the literature. In contrast, to make the integration of the OFS-based identification unit as a possible opportunity in an automated laboratory, we propose to set fixed (unique or eventually multiple) detection time. This alternative approach implies that the bacteria colonies to be identified can vary in dimensions. In the considered experiments the incubation time was chosen equal to 16 hours. This value allows the investigated bacteria species to reach a dimension in the range suitable for the scattering acquisition other than being an interesting value from a clinical point of view in that it slightly anticipates typical reading times used by microbiologists, thus giving the possibility to devise some form of computer aided or enhanced diagnosis. To make the forward-scattering technique working, the laser beam diameter must be similar to the colony diameter; therefore, with our approach the laser beam diameter must be variable. In the prototype implementation the diameter of the laser beam can be 
manually set, in a possible actual implementation the diameter adjustment will be automated. This can be facilitated by the fact that both the colony centering and the laser beam diameter adjustment can rely on the vision system used to acquire plate images. The presented preliminary experimental activities have shown that the colony dimension variability influences both the pattern dimension and the pattern features which are equally strictly depending on the colony growing phase reached at the moment of the image acquisition.

As the scattering angle is related to the colony dimension and diameter, we suggest including the possibility of changing the camera distance from the Petri dish. As the colony dimension increases the scattering pattern shrinks, the movement of the camera can be used to compensate the image shrinking maintaining a constant relative image resolution. Moreover we could define an acquisition of scattering pattern sequences composed by images from different camera distances.

In the full automated lab the analysis of the OFS patterns should be demanded to a proper image processor implementing algorithms able to discriminate the images coming from the different pathogens. In this frame, the repeatability of the OFS patterns observed in the experiments allows us to be confident that reliable and efficient image processing algorithms could be successfully applied.

Finally, some considerations about the risks related to the application of the OFS technology in the automated labs. Despite the discriminative power of the OSF images is proven for recognizing different pathogens, the selection of the image analysis tools could be critical. Statistical machine learning approaches seems to be particularly suitable due their capability of classification with a likelihood information which would be of great value for risk analysis procedures. The classification algorithms may be calibrated in order to be cautious and conservative when searching for pathogens for which a wrong identification would have severe consequences. The risk reduction could also benefit from redundancy obtained by the acquisition of images from more than one colony from the same plate. The redundancy policy could be defined by an appropriate risk analysis. In any case, we think the OFS technique is best suited to implement a presumptive bacteria identification system collaborating with the reliable current standard techniques. In light of this, the risk analysis can be used to define to what extent the OSF technology contributes to the overall improvement of the timing and quality of the diagnostic processes in an enriched multimodal system for bacterial identification. 
Moreover, from the above experimental observations related to the various acquisition setting variabilities, it can be suggested that a properly structured image database should be collected for an optimal training of either specialized microbiologists or machine learning techniques. For example it will be meaning to acquire pattern images referring to the different colony growing phases, that is, patterns corresponding to different colony dimensions. In this way, provided that during the classification it should be possible to estimate the colony growing phase, the classification algorithms can identify the bacteria species relying on how information is structured and labeled in the reference image database.

\section{Conclusion}

In view of the possible application of the forward-scattering technique in the automated clinical analysis laboratory we have examined the limiting factors of the current implementations of the technique. We proved that the fixed colony diameter constraint is incompatible with the envisaged application and therefore it is necessary to make the application of the forward-scattering technique more flexible. In this prospect, we propose to acquire the scattering images after a single or a multiple predetermined incubation duration since this is the condition that best matches the requirements from the possible integration of the scattering unit into an automated specimen processing workflow.

From the experimental activity on a selected set of bacteria (UTI) we can say that the constraint of fixed time incubation can be considered compatible with the scatteringbased identification technique provided that the acquisition unit is able to adjust, in realtime, its hardware configuration to adapt the pattern acquisition to the growing phase of the investigated colony. Moreover, the reference images database must be filled with pattern images of known bacteria captured at different growing phases. The main elements of the scatterometer involved in the new design are the optical source and the optics and positioning of the scattering image acquisition camera. The proposed solution of automatic beam diameter adjustment and camera positioning are well compatible with modern automation setups. Also important for an effective implementation is the definition of a procedure to roughly estimate the growing phase of the selected colony at the moment of the scattering pattern acquisition. 


\section{Acknowledgments}

We thank Copan Italia S.p.a. for the vital support for the realization of this study. A special thanks to the late Dr. Roberto Vaiani for his passion and enthusiasm in dealing with microbiology and to Dr. Santina Castriciano for the helpful insights. 


\section{References}

Akova, F., Dundar, M., Davisson, V. J., Hirleman, E. D., Bhunia, A. K., Robinson, J. P., Rajwa, B., 2010. Stat. Anal. Data Min. 3, 289-301.

Bae, E., Banada, P. P., Huff, K., Bhunia, A. K., Robinson, J. P., Hirleman, E. D., 2007. Appl. Opt. 46, 3639-3648.

Bae, E., Aroonnual, A., Bhunia, A. K., Robinson, J. P., Hirleman, E. D., 2009. Meas. Sci. Tech. 20, 0158021-0158029.

Bae, E., Bai, N., Aroonnual, A., Robinson, J. P., Bhunia, A. K., Hirleman, E. D., 2010. J. Biomed. Opt. 15, 045001_1-045001_11.

Bae, E., Aroonnual, A., Bhunia, A. K., Hirleman, E. D., 2011a. J. Biophotonics. 4, 236-243.

Bae, E., Bai, N., Aroonnual, A., Bhunia, A. K., Hirleman, E. D., 2011b. Biotechnol. Bioeng. 108, 637-644.

Banada, P. P., Guo, S., Bayraktar, B., Bae, E., Rajwa, B., Robinson, J. P., Hirleman, E. D., Bhunia, A. K., 2007. Biosens. Bioelectron. 22, 1664-1671.

Banada, P. P., Huff, K., Bae, E., Rajwa, B., Aroonnual, A., Bayraktar, B., Adil, A., Robinson, J. P., Hirleman, E. D., Bhunia, A. K., 2009. Biosens. Bioelectron 24, 1685-1692.

Bourbeau, P. P., Ledeboer, N. A., 2013. J. Clin. Microbiol. 51, 1658-1665.

Buzalewicz, I., Wieliczko, A., Podbielska, H., 2011. Opt. Expr. 19, 21768-21785.

Gong, H., Chen, B., Zhang, X., Tseng, C. C., 2013. IJCBDD 6, 234-243.

Khotanzad, A., Hong, J. H., 1990. IEEE Trans. Pattern Anal. Mach. Intell. 12, 489-497.

Novak, S. M., Marlowe, E. M., 2013. Clin. Lab. Med. 33, 567-588.

Patel, R., 2013. Clin. Infect. Dis. 57, 564-572.

Rajwa, B., Bayraktar, B., Banada, P. P., Huff, K., Bae, E., Hirleman, E. D., Bhunia, A. K., Robinson, J. P., 2006. SPIE 6381, 6381051-6381058.

Risch, M., Radjenovic, D., Han, J. N., Wydler, M., Nydegger, U., Risch, L., 2010. Swiss Med Wkly. 140, w13095.

Seng, P., Drancourt, M., Gouriet, F., La Scola, B., Fournier, P. E., Rolain, J. M., Raoult, D., 2009. Clin. Infect. Dis. 49, 543-551.

Suchwalko, A., Buzalewicz, I., Wieliczko, A., Podbielska, H., 2013. Opt. Exp. 21, 11322-11337.

Yoon, S. C., Lawrence, K. C., Line, J., Siragusa, G., Feldner, P., Park, B., Windham, W. R., 2010.

Sens. \& Instrumen. Food Qual. 4, 35-49.

Yoon, S. C., Windham, W. R., Ladely, S., Heitschmidt, G. W., Lawrence, K. C., Park, B., Narang, N., Cray, W. C., 2013. J. Food Meas. Charact. 7, 47-59. 